\title{
Experimental Study of Longitudinal and Circumferential External Defect in Pressured Cylindrical Shells
}

\author{
A. Elhakimi1 , A. Chamat ${ }^{2}$, A. Touache1, A. Daya ${ }^{3}$, S. Hariri ${ }^{4}$ \\ ${ }^{1}$ Département Génie Mécanique, Faculté des Sciences et Techniques de Fès, Fès, Maroc \\ ${ }^{2}$ Département Génie Industriel, Faculté des Sciences et Techniques de Fès, Fès, Maroc \\ ${ }^{3}$ Département de Physique, LMMESA, Faculté des Sciences et Techniques Errachidia, Errachidia, Maroc \\ ${ }^{4}$ Département Technologie des Polymères et Composites and Ingénierie Mécanique, \\ Ecole des Mines de Douai, Douai, France \\ Email: ${ }^{*}$ chamat.abderrahim@yahoo.fr
}

Received 19 April 2015; accepted 1 June 2015; published 4 June 2015

Copyright (C) 2015 by authors and Scientific Research Publishing Inc.

This work is licensed under the Creative Commons Attribution International License (CC BY).

http://creativecommons.org/licenses/by/4.0/

c) (i) Open Access

\begin{abstract}
Two cylindrical vessels under internal pressure are used for this work in order to study the influence of the position and size of defects on their elastic and elastoplastic behavior. One contains two external longitudinal semi-elliptic defects of different dimensions realized diametrically opposed. The other contains the same defects but is circumferential. These defects are carried out by elect-erosion. Strain gauges are placed in the neighborhood of the defects of which the purpose is to obtain the strain distribution. This work also allows the comparison between two defects of different dimensions, which are of the same shape or different shapes. These defects are longitudinal and circumferential semi-elliptical. The position of these defects relative to the inner radius of a cylindrical pressure vessel is considered. The deformations results are discussed.
\end{abstract}

\section{Keywords}

Cylindrical Shell under Pression, Longitudinal Defect, Circumferential Defect, External Defect, Crack, Elastic Deformation

\section{Introduction}

In industrial structures, pressure vessels have been a major subject of research for several decades: gas transfer

"Corresponding author.

How to cite this paper: Elhakimi, A., Chamat, A., Touache, A., Daya, A. and Hariri, S. (2015) Experimental Study of Longitudinal and Circumferential External Defect in Pressured Cylindrical Shells. World Journal of Mechanics, 5, 95-105.

http://dx.doi.org/10.4236/wjm.2015.56011 
pipelines and oil [1]-[4], tank. The characterization of the harmfulness of this type of defects is fundamental to know, among others, the residual life of cracked structures. Consequently, the procedures of the rupture estimations for components under pressure play a key role [5]-[7] in the safety of these devices. All these robust procedures become necessary to specify the end of critical defect size that can take remedial decisions and an extension of the life of the components of the service structure. Our work consists in the study of the deformation evolution at the bottom and lip defects in pressure vessels. We are interested in the problems of semi-elliptical external defects in cylindrical shells. The experimental study was performed using two models of pressure vessels, designed according to the recommendations CODAP [8].

In the first geometry, we make two semi-elliptical longitudinal defects diametrically opposed by the process electron discharge machining, while the second model contains two circumferential defects diametrically opposite. These defects are instrumented with strain gauges to measure deformations. In this paper, we study four semi-elliptic defects: two longitudinal and two circumferential. To deduct the most critical between longitudinal defects and circumferential, we compare their openings deformations in the case of a purely elastic behavior.

\section{Experimental Study of a Cylindrical Shell}

\subsection{Characterization of the Studied Models}

The experimental study is performed on models consist of a cylindrical shell closed by two semi spherical fund large square radius (Figure 1). The external and internal diameters are $406.4 \mathrm{~mm}$ and $386.4 \mathrm{~mm}$, respectively

\subsection{Characterization of the Studied Material}

To determine the mechanical characteristics of the material studied, we realized tensile tests at room temperature. The test specimens were made in the longitudinal direction of the cylinder. We performed 6 cylindrical specimens according to standard NF A 03-172 [9], of circular cross section of diameter $7 \mathrm{~mm}$. The shape and dimensions of the specimens are shown in Figure 2.

Figure 3 represents the experimental conventional curve of the evolution of the stress as a function of the material's deformation. The general shape of this curve shows a ductile material behavior. The main mechanical characteristics of the material obtained by the tensile tests are summarized in Table 1 . The chemical composition of the material was determined by spectroscopy. The weight contents of the addition of the material elements [10] are in accordance with the P264GH steel.

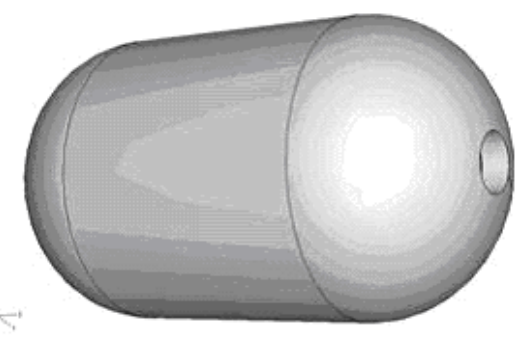

Figure 1. Geometry of the model (view the whole model).

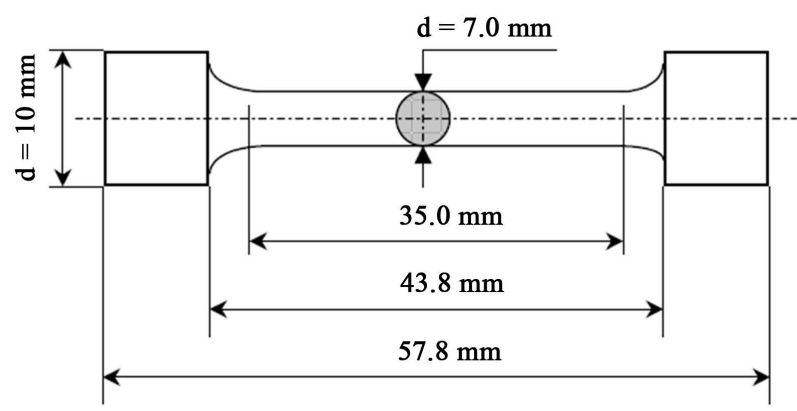

Figure 2. Shape and dimension of tensile specimens. 


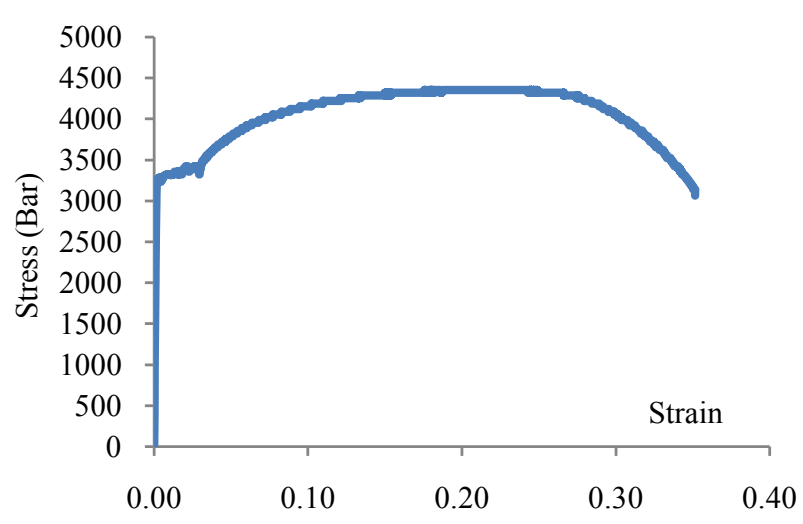

Figure 3. Tensile curve.

Table 1. Mechanical characteristics of the material models.

\begin{tabular}{ccccc}
\hline \multicolumn{4}{c}{ Mechanical characteristics } \\
\hline Young's modulus (bars) & Poisson's coefficient & Yield strength at $0.2 \%$ (bars) & Tensile strength (bars) & Elongation (A \%) \\
\hline $2,070,000$ & 0.3 & 3400 & 4400 & 35 \\
\hline
\end{tabular}

Testing, pressures in the pressure vessels have been made within the School of Mines de Douai Mechanics Laboratory according to standard CODAP.

\subsection{Realization of Defects}

In the experimental part, the studied defects are supposed semi-elliptical through-in outer surface, circumferential or longitudinal. We note "a" the depth of the defect and "2ce" their length. Each model includes two diametrically opposed defects of different sizes, so as to exploit maximum results on testing a minimum. Both defects are far enough from each other so as not to influence each other. The first cylindrical enclosure contains two longitudinal defects (Figure 4).

In the second cylindrical enclosure, the two defects are circumferential semi-elliptical in Figure 5, whereas Table 2 summarizes the various dimensions and orientations of defects in each model.

The defects are realized so as to have the same ratio a/c equal to $1 / 4$.

The cylindrical enclosure instrumented strain gauges, chain type, is put under internal pressure so that the objective is to follow the evolution of the deformations in zones near to defects. These defects are considered as notch [11] [12]. These chains contain each 5 circumferential or longitudinal gauges which were glued in zones closest defects.

\subsection{Instrumentation of Models}

\subsubsection{By Strain Gauges}

Chains are placed in the model (Figure 6), to determine experimentally the strain distribution in the neighborhood of the defects (in the elastic domain).

This cylindrical enclosure is instrumented with strain gauges according to Figure 7.

D1 and D2 are longitudinal defects whereas D3 and D4 are circumferential defects. Near the D1 longitudinal default, we stuck three chains: The chain $\mathrm{C}_{1}$ consisting in the first circumferential gauges, is located at the end of the defect and the two others (one consisting in circumferential $\mathrm{C}_{2}$ gauges and the other longitudinal in gauges $\mathrm{C}_{3}$ ) are in the middle of the defect. While in neighborhood of the longitudinal defect D2, two chains gauges are glued, the first $\mathrm{C}_{4}$ placed in the middle of the defect, measuring longitudinal deformations and the second $\mathrm{C}_{5}$ measures the longitudinal strains at the bottom of the defect.

For the circumferential defect D3, four chains are stuck: two chains at the end of the defect, the first chain $\mathrm{C}_{1}$ consisting in circumferential gauges and the second $\mathrm{C}_{4}$ consists in longitudinal gauges and the other two chains (one constituted of longitudinal gages $\mathrm{C}_{2}$ and the other circumferential gauges $\mathrm{C}_{3}$ ) in the middle of the defect. 

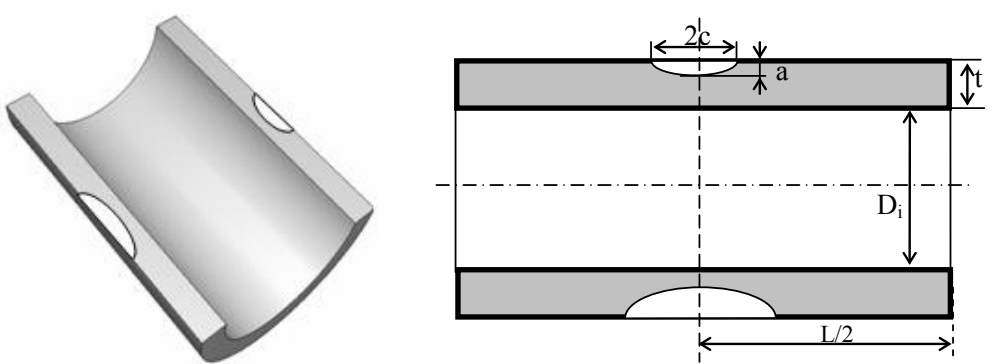

Figure 4. Position of the external semi-elliptical longitudinal defects
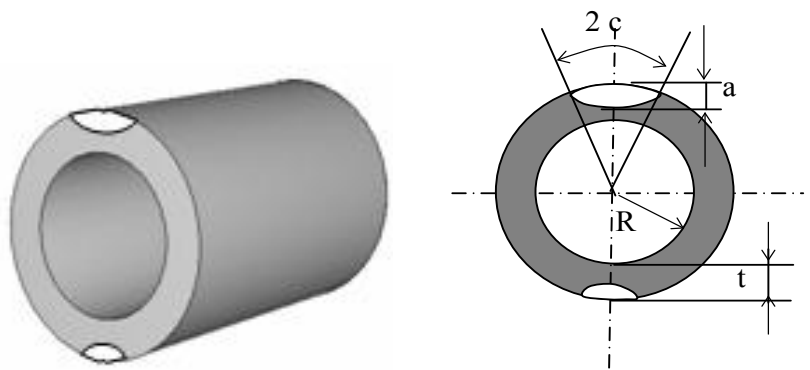

Figure 5. Position of the external semi-elliptical circumferential defects.

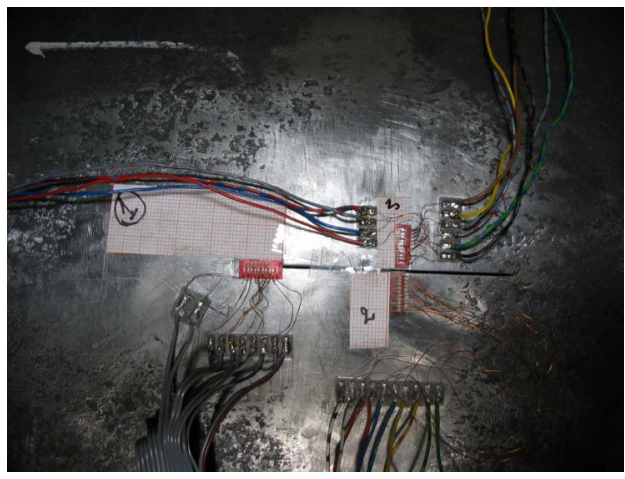

Instrumentation gauges (large defect)

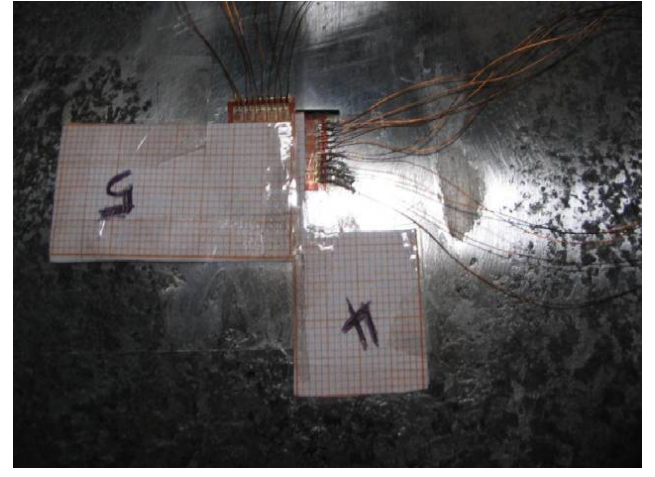

Instrumentation gauges (small defect)

Figure 6. Location meadows gauges defects.

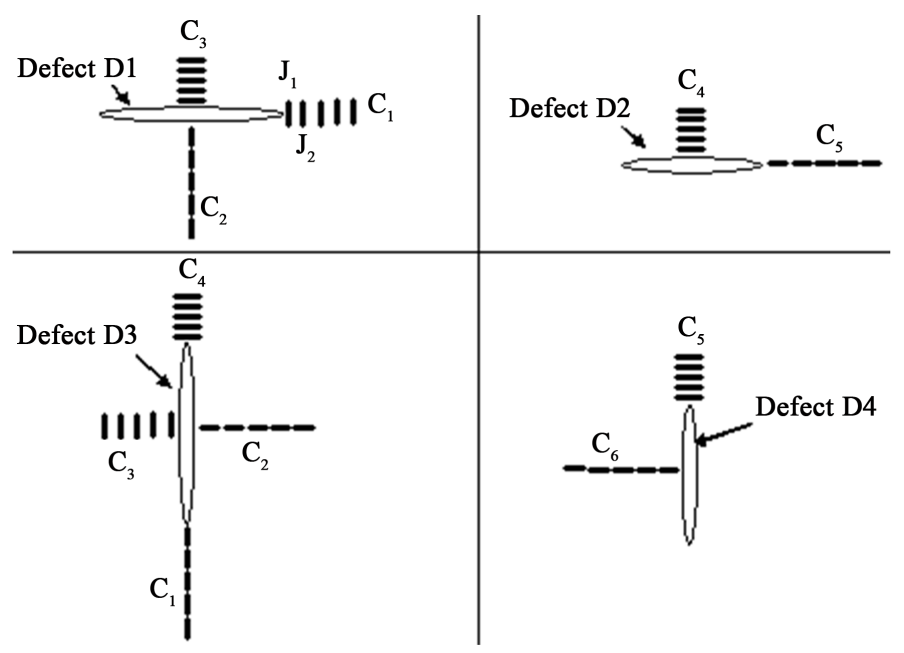

Figure 7. Position of chains in the neighborhood of the defects. 
Table 2. Geometric characteristics of defects in both models.

\begin{tabular}{cccc}
\hline Models & Defects & Orientations & Dimensions \\
M1 & $\mathrm{D}_{1}$ & longitudinal & $\mathrm{a}=8 \mathrm{~mm}$ \\
$\mathrm{c}=32 \mathrm{~mm}$ & $\mathrm{a}=2 \mathrm{~mm}$ \\
& $\mathrm{D} 2$ & longitudinal & $\mathrm{c}=8 \mathrm{~mm}$ \\
& & & $\mathrm{a}=8 \mathrm{~mm}$ \\
$\mathrm{c}=32 \mathrm{~mm}$ \\
\end{tabular}

While for the circumferential defect $\mathrm{D} 4$, we placed two chains: the first $\mathrm{C}_{5}$ consisting in longitudinal gauges is located at the end of the defect and the second $\mathrm{C}_{6}$ allows also measure the longitudinal deformations.

\subsubsection{By Acoustic Emission Sensor}

In order to confirm results obtained by the strain gauges, the resonant piezoelectric sensors are placed near defects (Figure 8). The amplitude of the resonance peak should not vary by more than $3 \mathrm{~dB}$ for a lot of sensors used in a same consideration of pressure equipment. The preamplifier gain is regulated in general to a range of amplification of $40+/-10 \mathrm{~dB}$ with a filtration system adapted to the sensor response. The choice of sensors is based on the CEN pr EN 13477 standard [13] for metal pressure equipment.

The dimension of the cylindrical shell comply with the General rules of construction of CODAP, we choose a $\mathrm{D}$ category of construction. The nominal stress of calculation is $\sigma_{f}=1470 \mathrm{bars}$. The pressure of the cylindrical shell is obtained by inverting the formula C2.1.4.2 CODAP:

$$
p=\frac{2 \times \sigma_{f} \times t \times z}{D_{m}}
$$

With $D_{m}, \sigma_{f}, t, z$ are respectively the mean diameter of the envelope, the nominal stress of calculation of the envelope's material, the thickness of the envelope and welding coefficient (as the ring is taken in seamless tube, it will take $z=1$ ).

The numerical application, of this equation and considering the previous data, gives a $\mathrm{P}$ pressure 74 bars. Pressure testing models, were performed in the laboratory of mechanical engineering from the Ecole of the Mines of Douai with water as pressure fluid. To realize the acoustic emission testing of pressure equipment, the applied pressure must be higher than the applied maximum pressure (AMP) in service (the recommended pressure is $110 \%$ of AMP). The pressurization cycles are carried out in accordance with the recommendation of the GBP [14] (Figure 9).

The various stages of solicitations of specimens are:

$>$ Fill in water the specimen until the admissible maximum height;

$>$ Load the structure (mean velocity of pressure rise of $0.033 \mathrm{bars} / \mathrm{s}$ ) and descent is done at a speed of 0.033 bars/s too;

The load must be maintained for a minimum period of ten minutes.

\subsection{Determination of the Elastic Pressure Experimentally}

\subsubsection{By Strain's Gauges}

In order to work in the elastic domain, a cylindrical shell was loaded by an internal pressure $\mathrm{p}$ and the circumferential strain $\varepsilon_{\theta \theta}$ was measured in the neighborhood of the longitudinal defect D1. Figure 10 shows the evolution of the pressure (in bar) versus the circumferential deformation $\varepsilon_{\theta \theta}$ registered by $\mathbf{J}_{\mathbf{1}}$ and $\mathbf{J}_{\mathbf{2}}$ gauges of the chain $\mathrm{C}_{1}$ located respectively in $2 \mathrm{~mm}$ and $4 \mathrm{~mm}$ of the edge of longitudinal defect D1. We noted the plasticization of the material in $\mathbf{J}_{\mathbf{1}}$ before $\mathbf{J}_{\mathbf{2}}$ those is due to the stress concentrations at the bottom of the defect. The different elastic limit raised by $\mathbf{J}_{\mathbf{1}}$ and $\mathbf{J}_{2}$ is respectively $1108 \mu$-strains and $766 \mu$-strains. Whereas in the zone of deformation the most requested in the neighborhood of defect passes in the plastic range to 40 bars in $\mathbf{J}_{1}$ and 42 bars in $\mathbf{J}_{\mathbf{2}}$. This proves that the material is plasticized initially at the bottom of the defect. 


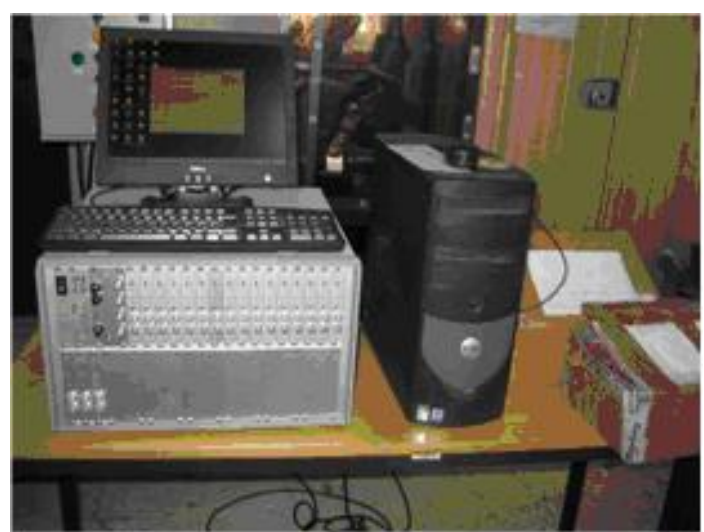

Measuring equipment

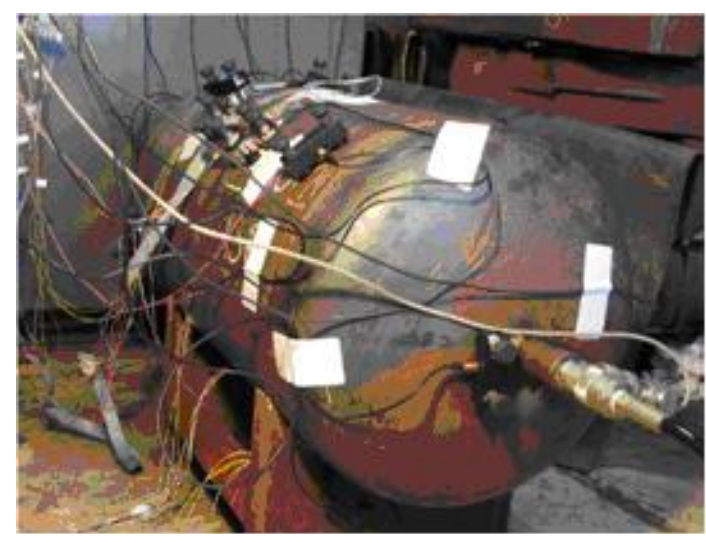

Specimen with gauges and sensors

Figure 8. Acquisition chain of signal.

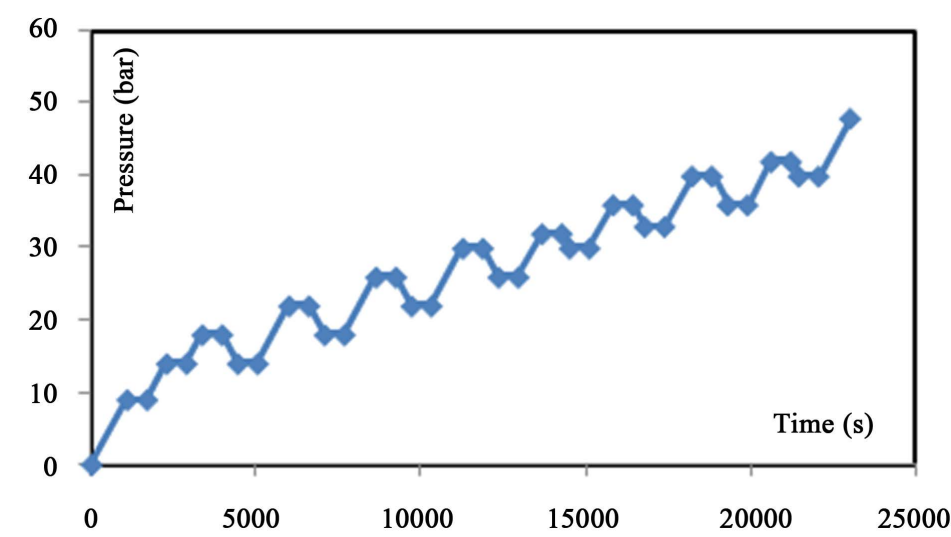

Figure 9. Example of pressure cycle.

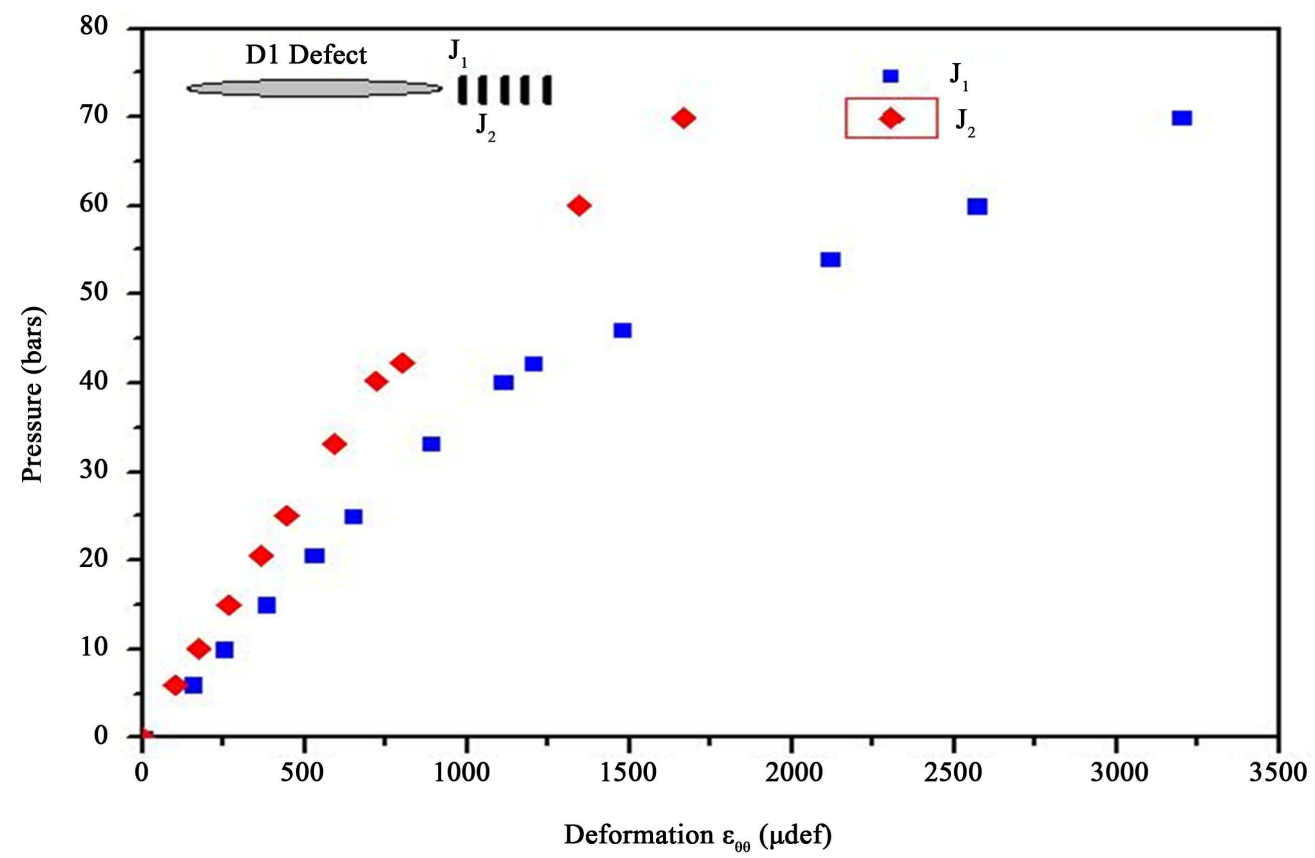

Figure 10. Evolution of deformation $\varepsilon_{\theta \theta}$ as function of pressure $\mathrm{P}$ in $\mathrm{J}_{1}$ gauge and $\mathrm{J}_{2}$ gauge of $\mathrm{D} 1$ defect. 


\subsubsection{By Acoustic Emission}

Figure 11 represents the record of acoustic emission based on time of the second model, according to the cycle of pressure, for each the sensors.

After a silent period, the acoustic emission gradually changes according to the rise in pressure. A pressure of 40 bars, the acoustic activity becomes more important. What Attests the beginning of plasticization in the neighborhood of the defects and confirms the results obtained by gauges deformations.

So that the various cylindrical shells go an elastic behavior, we have chooses a pressure of 25 bars in order to be sure that our test-tubes do not undergo any plastic deformation and they behave elastically.

\section{Experimental Study of the Distribution of Deformation in Different Cylindrical Vessels under Pressure}

The internal pressure is constant during all the tests and is equal to 25 bars. We chose the same geometrical reports of the defects retained in the experimental part of two defects namely: $\mathbf{a} / \mathbf{t} ; \mathbf{t} / \mathbf{R}_{\mathbf{i}} ; \mathbf{c} / \mathbf{a}$ (Figure 4).

\subsection{Study of the Behavior of the First Cylindrical Vessels Carrying Two Longitudinal Defects}

The deformations raised by the various chains are represented in Figure 12, and $\mathrm{x}$ is the position of the gauge relative to the bottom of the crack or relative to the lip of the crack. We note that the circumferential deformation relieved by $\mathrm{C}_{1}$ chain is larger at the bottom of the defect than relieved by $\mathrm{C}_{2}$ chain in the middle of the lip of

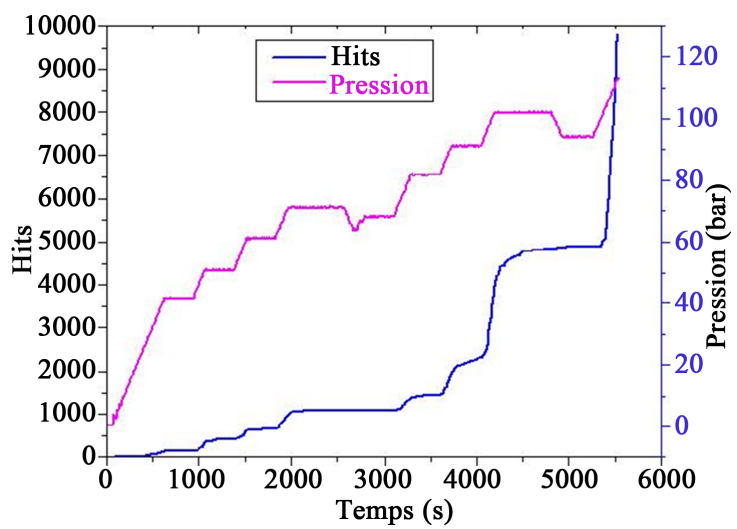

Sensor 1

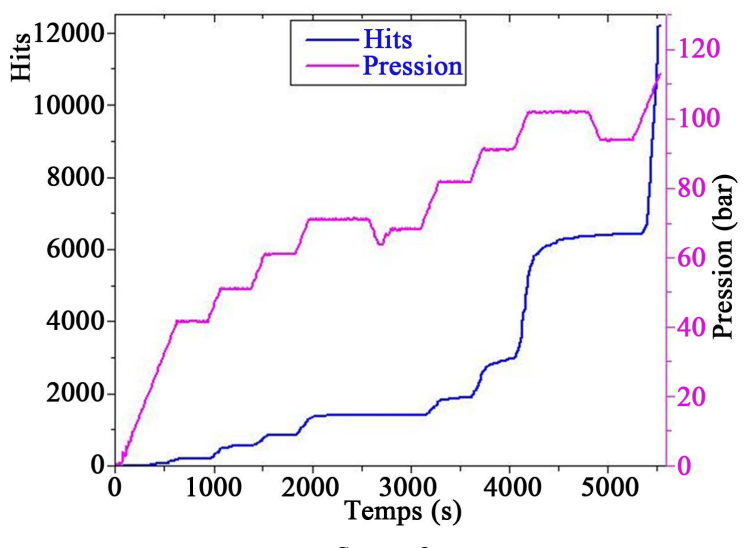

Sensor 2

Figure 11. Evolution of Hits as a function of time according to the pressure cycle (Hits: cumulative number of acoustic events).

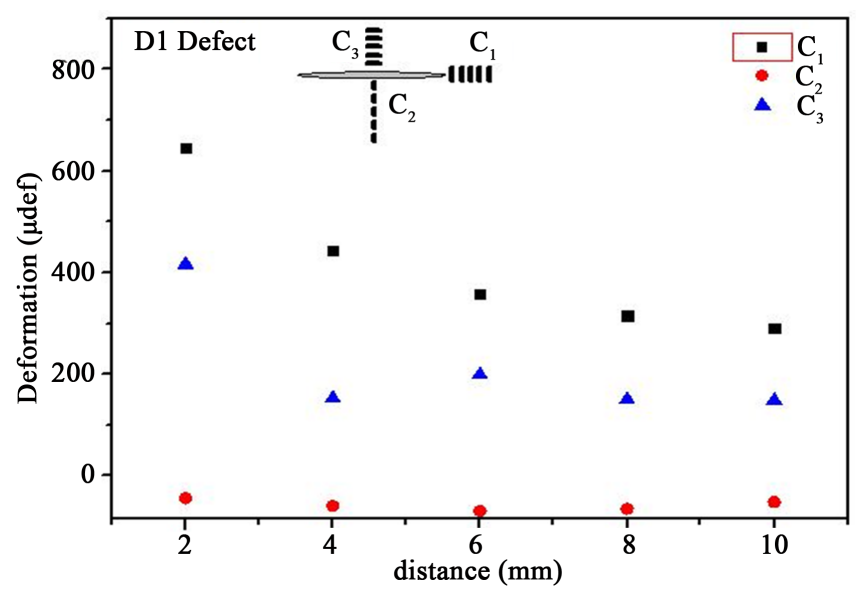

Figure 12. Evolution of the deformation of the $C_{1}, C_{2}$ and $C_{3}$ chains of the D1 defect. 
the defect. The positive deformation at the bottom of the defect shows its opening, whereas the negative circumferential deformation shows its compression on the level of lips. The longitudinal deformation measured by the $\mathrm{C}_{3}$ chain shows the lip is requested in traction along the first cylindrical vessels. Considering the three curves of deformation, we confirm that the circumferential deformation is the one which controls the opening of the defect. The maximal value of $644 \mu \mathrm{def}$ is reached at the bottom of the defect.

We compare the longitudinal deformations taken with the free lips of both D1 and D2 defects measured with the $\mathrm{C}_{3}$ and $\mathrm{C}_{4}$ chains of the same a/c (Figure 13). The D1 defect is to $2 \mathrm{~mm}$ of internal radius whereas the D2 defect is a little far from the internal radius to $8 \mathrm{~mm}$. This shows that the $\mathrm{D} 2$ defect is requested less than the D1 defect.

We noted that the longitudinal strain at the lip of defect is almost the same for both defects from $\mathbf{J}_{\mathbf{3}}$ gauge. The middle of the D2 lip is almost free and has undergone a deformation of $24.79 \mu$-strains, whereas the middle of the D1 lip underwent a deformation of $416 \mu$-strains. This shows that a/c report is not only significant, but it is necessary to specify moreover the position of the semi-elliptical defect from the internal radius of the cylindrical enclosure.

\subsection{Study of the Behavior of the Second Cylindrical Enclosure Carrying Two Circumferential Defects}

In this cylindrical enclosure, we carried out two semi-elliptical external defects diametrically opposite. The chains $\mathrm{C}_{2}$ and $\mathrm{C}_{4}$ measure the longitudinal deformation, whereas the chains $\mathrm{C}_{1}$ and $\mathrm{C}_{3}$ measure the circumferential deformation (Figure 14).

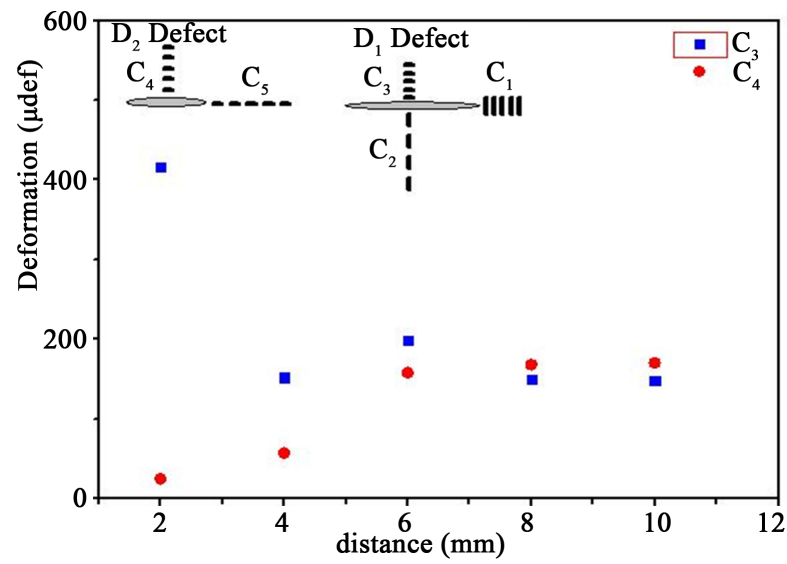

Figure 13. Evolution of the longitudinal strain of the $\mathrm{C}_{3}$ chains of the D1 defect and the $\mathrm{C}_{4}$ chains of the D2 defect.

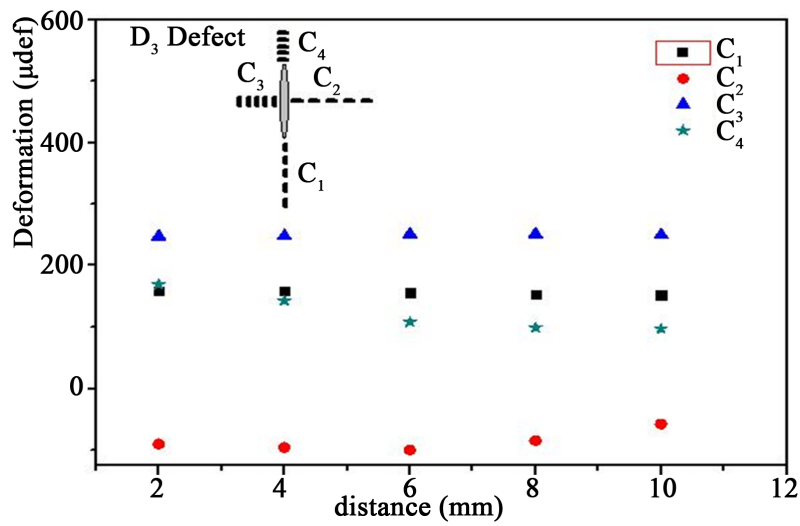

Figure 14. Evolution of the deformation of the $C_{1}, C_{2}, C_{3}$ and $C_{4}$ chains of the D3 defect. 
We note that the maximum circumferential deformation, $247 \mu$-strain, is recorded by the $\mathrm{C}_{3}$ chain in the middle on the lip of the D3 defect. It is also followed by a circumferential deformation raised by the $\mathrm{C}_{1}$ chain. The other lip of the defect records a compression in its middle. We can conclude that the circumferential strain controls as much for the semi-elliptical circumferential defects as for the semi-elliptical longitudinal defects. Let us compare now the evolutions of the deformation between the D3 and D4 defects and check the influence of the position of the defect relative to the internal radius of the second cylindrical enclosure. For this, we will compare between the deformation raised by the $\mathrm{C}_{2}$ and $\mathrm{C}_{4}$ chains of the $\mathrm{D} 3$ defect and those raised by the $\mathrm{C}_{5}$ and $\mathrm{C}_{6}$ chains of the D4 defect and the evolution by finite element [7] (Figure 15 and Figure 16).

We note that the lip of D3 defect is under compression, whereas the lip of D4 defect is under traction. The defect area should be finely refined including special elements at the crack tip. A fundamental step is to calibrate and optimize the mesh. For this application is the CASTEM software that is used. But the simulation by the finite element method has shown that the two strains meet beyond the zone of disturbance.

The longitudinal deformation at the bottom of the two defects represents an extension of the cylindrical enclosure. The D3 defect is less sought with the disturbance, because it is to $8 \mathrm{~mm}$ of the internal radius of the cylindrical enclosure and the maximum strain is of $169 \mu$-strains. While the D4 defect is to $2 \mathrm{~mm}$ and the maximum strain is of $190 \mu$-strains. Table 3 recapitulates the deformation raised by the first gauge of each chain.

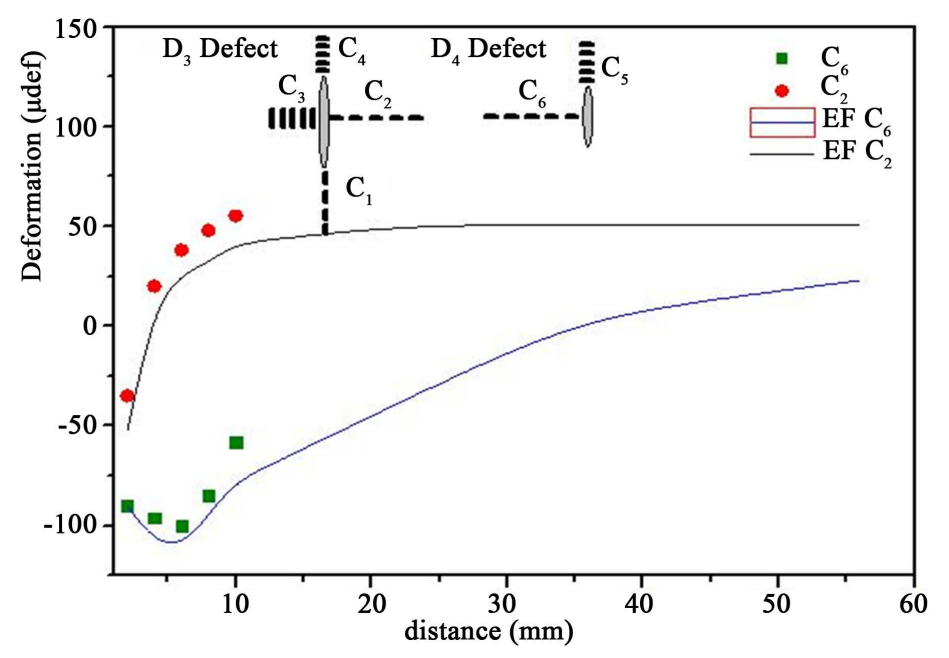

Figure 15. Evolution of the longitudinal deformation of the $\mathrm{C}_{2}$ chains of the D3 defect and the $\mathrm{C}_{6}$ of the $\mathrm{D} 4$ defect thus by finite element method.

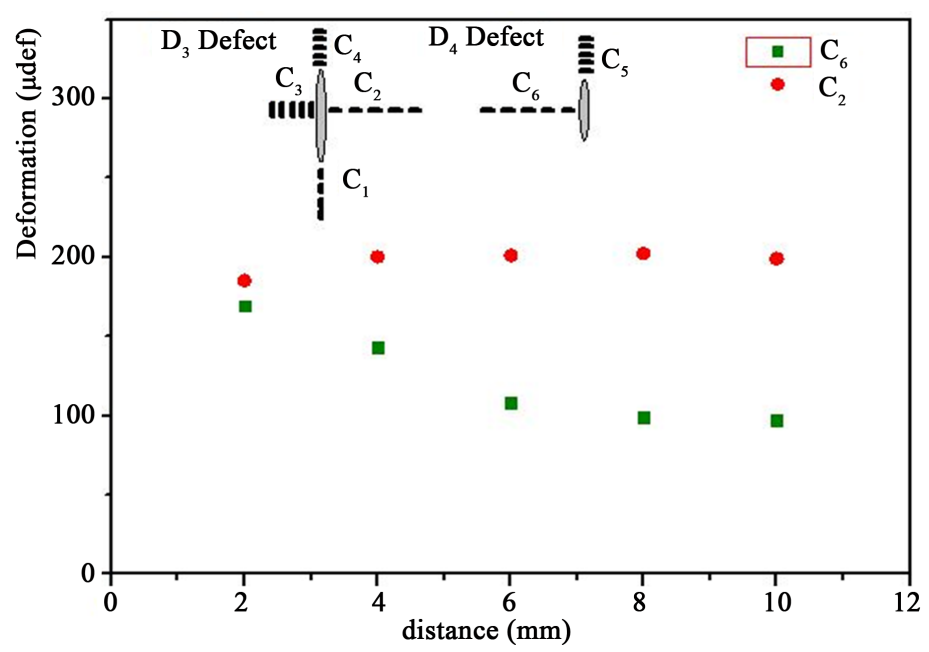

Figure 16. Evolution of the longitudinal deformation of the $\mathrm{C}_{4}$ chains of the D3 defect and the $\mathrm{C}_{5}$ of the D4 defect. 
Table 3. The deformation given by the $\mathrm{J}_{1}$ gauges of each chain.

\begin{tabular}{|c|c|c|c|c|c|c|}
\hline & $\mathrm{C}_{1}(\mu$-strain $)$ & $\mathrm{C}_{2}$ ( $\mu$-strain $)$ & $\mathrm{C}_{3}$ ( $\mu$-strain) & $\mathrm{C}_{4}(\mu$-strain $)$ & $\mathrm{C}_{5}(\mu$-strain) & $\mathrm{C}_{6}(\mu$-strain $)$ \\
\hline D1 defect & 644 & -45 & 416 & & & \\
\hline D2 defect & & & & 25 & 55 & \\
\hline D3 defect & 159 & -90 & 247 & 169 & & \\
\hline D4 defect & & & & & 185 & -35 \\
\hline
\end{tabular}

We note that the higher deformation is raised by the $\mathbf{J}_{2}$ gauge of the $\mathrm{C}_{1}$ chain of the D1 defect which measures a circumferential deformation at the bottom of the defect. At the same dimension, the D3 circumferential defect is requested less than the D1 longitudinal defect. For two defects of the same form (either longitudinal or circumferential) but of different dimensions, it is necessary to also take into account the position of these defects relative to the internal radius of the cylindrical enclosure. Regardless of the form of the defect; it is the deformation of circumferential opening which it is necessary to take into account for any use.

\section{Conclusion}

An experimental study, on cylindrical shells carrying of the semi-elliptical defects longitudinal and circumferential, was performed by using strain gauges of the neighborhoods of defect. We made the comparison between two semi-elliptical defects of the same dimension but one of them was oriented longitudinally and the other was circumferential. These defects are found in two different positions relative to the internal radius from the cylindrical enclosure. We noted that the longitudinal semi-elliptic defect is more dangerous than the circumferential through the study of circumferential opening deformation. A comparison among the strains has shown that it is the circumferential deformation at the bottom of the defect which constitutes the maximum. This work is of interesting value; it will allow us to study the effect of the harmfulness of cylindrical shells cracked with energy methods, the influence of the position and size of the cracks compared with the inner side pressure cup.

\section{References}

[1] Capelle, J., Gilgert, J., Dmytrakh, I. and Pluvinage, G. (2010) The Effect of Hydrogen Concentration on Fracture of Pipeline Steels in Presence of a Notch, Engineering Fracture Mechanics.

[2] Hadj Meliani, M., Azar,i Z., Pluvinage, G. and Capelle, J. (2010) Gouge Assessment for Pipes and Associated Transferability Problem. Engineering Failure Analysis, 17, 1117-1126. http://dx.doi.org/10.1016/j.engfailanal.2010.01.007

[3] Capelle, J., Gilgert, J. and Pluvinag, G. (2010) A Fatigue Initiation Parameter for Gas Pipe Steel Submitted to Hydrogen Absorption. International Journal of Hydrogen Energy, 35, 833-884.

[4] Jasper, A. (2012) Oil/Gas Pipeline Leak Inspection and Repair in Underwater Poor Visibility Conditions: Challenges and Perspectives. Journal of Environmental Protection, 3, 394-399. http://dx.doi.org/10.4236/jep.2012.35049

[5] Li, T.P. and Tian, X.L. (2013) Effect Analysis of Gurson Model Parameters on Crack Extension of Pipeline. Journal of Applied Mathematics and Physics, 1, 54-56. http://dx.doi.org/10.4236/jamp.2013.16011

[6] Ibrahim, A., Ryu, Y. and Saidpour, M. (2015) Stress Analysis of Thin-Walled Pressure Vessels. Modern Mechanical Engineering, 5, 1-9. http://dx.doi.org/10.4236/jamp.2013.16011

[7] Arab, N. and Javadimanesh, A. (2013) Theoretical and Experimental Analysis of Deep Drawing Cylindrical Cup. Journal of Minerals and Materials Characterization and Engineering, 1, 336-342. http://dx.doi.org/10.4236/jmmce.2013.16051

[8] CODAP (Code de construction des appareils à pression) (2005) SNCT Publications.

[9] Standard NF A 03-172 (1980) Produits sidérurgiques, valeurs de conversion de dureté de l'acier.

[10] El Hakimi, A. (2006) Etude numérique et expérimentale de la nocivité des defaults dans des coques cylindriques et sphériques sous pression. Thèse de l'université de technologie de Compiègne Juin.

[11] Moustabchir, H., Azari, Z., Hariri, S. and Dmytrakh, I. (2010) Experimental and Numerical Study of Stress-Strain State of Pressurised Cylindrical Shells with External Defects. Engineering Failure Analysis, 17, 506-514. http://dx.doi.org/10.1016/j.engfailanal.2009.09.011

[12] Moustabchir, H., Azari, Z., Hariri, S. and Dmytrakh, I. (2012) Experimental and Computed Stress Distribution Ahead 
of a Notch in a Pressure Vessel: Application of T-Stress Conception. Computational Materials Science, 58, 59-66.

[13] CEN pr EN 13477 Standards Consists of the Following Parts under the General Title Non Destructive Testing-Acoustic Emission-Equipment Characterization.

[14] GBP-EA: Guide de Bonnes Pratiques pour le contrôle par Emission Acoustique des équipements sous pression, Association françaises des ingénieurs en appareils à pression (A.F.I.A.P.), édition 2001. 